\title{
Vision-based Target Geo-location Using a Fixed-wing Miniature Air Vehicle
}

D. Blake Barber

Brigham Young University - Provo

Joshua D. Redding

Scientific Systems Company, Inc., joshua@ssci.com

Timothy W. McLain

Brigham Young University - Provo, mclain@byu.edu

Randal W. Beard

Brigham Young University - Provo, beard@byu.edu

Clark N. Taylor

Brigham Young University - Provo

Follow this and additional works at: https://scholarsarchive.byu.edu/facpub

Part of the Mechanical Engineering Commons

\section{Original Publication Citation}

Barber, D., Redding, J., McLain, T., Beard, R., and Taylor, C. Vision-based Target Geo-location Using a Fixed-wing Miniature Air Vehicle, Journal of Intelligent and Robotic Systems, vol. 47, no. 4, pp. 361-382, December 2006.

\section{BYU ScholarsArchive Citation}

Barber, D. Blake; Redding, Joshua D.; McLain, Timothy W.; Beard, Randal W.; and Taylor, Clark N., "Visionbased Target Geo-location Using a Fixed-wing Miniature Air Vehicle" (2006). Faculty Publications. 1502. https://scholarsarchive.byu.edu/facpub/1502 accepted for inclusion in Faculty Publications by an authorized administrator of BYU ScholarsArchive. For more information, please contact ellen_amatangelo@byu.edu. 


\title{
Vision-based Target Geo-location using a Fixed-wing Miniature Air Vehicle
}

\author{
D. Blake Barber ${ }^{1}$, Joshua D. Redding ${ }^{2}$, Timothy W. McLain ${ }^{1}$, Randal W. Beard ${ }^{1}$, Clark N. Taylor ${ }^{1}$ \\ ${ }^{1}$ Magicc Lab, Brigham Young University, Provo, UT 84602 \\ ${ }^{2}$ Scientific Systems Company, Inc., Woburn, MA 01801
}

Submitted 24 August 2006

\begin{abstract}
This paper presents a method for determining the GPS location of a ground-based object when imaged from a fixed-wing miniature air vehicle (MAV). Using the pixel location of the target in an image, with measurements of MAV position and attitude, and camera pose angles, the target is localized in world coordinates. The main contribution of this paper is to present four techniques for reducing the localization error. In particular, we discuss RLS filtering, bias estimation, flight path selection, and wind estimation. The localization method has been implemented and flight tested on BYU's MAV testbed and experimental results are presented demonstrating the localization of a target to within 3 meters of its known GPS location.
\end{abstract}

Key words. Unmanned air vehicles, geo-location, computer vision, micro air vehicles, localization

\section{Introduction}

Unmanned air systems are prime candidates for tasks involving risk and repetition, or what the military calls the "dull, dirty and dangerous" (Office of the Secretary of Defense, 2002). For tasks that involve tracking, reconnaissance, and delivery, one objective of unmanned air systems is to accurately determine the location of ground-based objects.

This paper presents a method for determining the location of objects in world/inertial coordinates using a gimballed EO/IR camera on-board a fixed-wing miniature air vehicle (MAV). We focus on fixed-wing UAVs (as opposed to rotary wing aircraft or blimps) due to the unique benefits available from fixed-wing aircraft, including: adaptability to adverse weather, enhanced fuel efficiency, a shorter learning curve for the untrained operator, and extreme durability in harsh environments. Also, minimum airspeed requirements associated with fixed-wing aircraft can provide images from multiple vantage points, allowing for more robust localization.

Correspondence to: R. Beard

e-mail: beard@ee.byu.edu
In this paper we have assumed that the target is identified in the video stream by a human end user. The target is then automatically tracked at frame rate using a combination of color segmentation and feature tracking (Ma et al., 2003). After the object has been identified in the video stream and an initial estimate of its world coordinates has been determined, the MAV is commanded to orbit the object in order to collect additional information that is used to further enhance the estimate. Due to the specific nature of MAVs, there are several sources of error that affect the position estimates. In this paper, we analyze the error sources and present four steps to enhance the accuracy of the estimated target location.

While vision-based localization is well understood, previously published results focus on unmanned ground vehicles (Saeedi et al., 2003; Chroust and Vincze, 2003), or stationary air vehicles such as a blimps (Chaimowicz et al., 2004) or rotorcraft (Vidal and Sastry, 2002). However, blimps are not well suited for use in high winds or inclement weather, and the costs and complexities associated with rotorcraft are non-trivial. The objective of this paper is to explore localization methods using fixed-wing MAVs which tend to be more robust and less-expensive platforms.

Previous results on geo-locating objects from fixed-wing aircraft have several limitations not present in the system described in this paper. In (Kumar et al., 1998, 2000), all information collected by an aerial camera is accurately geolocated through registration with pre-existing geo-reference imagery. In contrast, our system focuses on geo-locating a specific object in the video stream and does not require preexisting geo-referenced imagery. A method for creating georeferenced mosaics from aerial video is presented in (Schultz et al., 2000), however, this method assumes an extremely accurate IMU that is impractical for MAVs due to weight and power restrictions.

Several previous works on target tracking/localization from UAVs are focused on control of the UAV to keep the object in view, as opposed to actually geo-locating the objects (Rysdyk, 2003; Stolle and Rysdyk, 2003; Wang et al., 2005; Frew and Rock, 2003). In (Rysdyk, 2003) flight paths for fixed-wing UAVs are designed to maintain a constant lineof-sight with a ground-based target. Stolle and Rysdyk (2003) present similar results with some useful details on camera control. Both references focus on pointing a UAV-mounted 
camera at a known target location and present simulation results. The accuracy of the localization results is not discussed.

The geo-location system presented in (Gibbins et al., 2004 ) is similar to our work, however, the reported errors are in excess of 20 meters, while our method achieves localization errors under 5 meters. Whang et al. (2005) and Dobrokhodov et al. (2006) describe a geo-location solution that is similar to the work presented in this paper. Range estimates in (Dobrokhodov et al., 2006) are obtained using a terrain model, and a nonlinear filter is used to estimate the position and velocity of moving ground based targets. Campbell and Wheeler (2006) also presents a vision based geolocation system that is similar to our solution. The estimation scheme proposed in (Campbell and Wheeler, 2006) is based on a square root sigma point filter and can handle moving objects. Bounds on the localization error are explicitly derived from the filter. However, the results presented in (Dobrokhodov et al., 2006) and (Campbell and Wheeler, 2006) both exhibit biases in the estimate, and neither paper addresses the sensitivity of the solution to heavy wind conditions. Early versions of the results appearing in this paper are presented in (Redding et al., 2006).

The remainder of the paper is organized as follows. In Section 2, we present the basic mathematics used to obtain the raw target localization estimates from a single frame within the video. In Section 3 we discuss four techniques for improving the localization estimate of the target. We present flight results demonstrating the effectiveness of our method in Section 4, and offer some concluding remarks in Section 5.

\section{The Geometry of Geo-location}

In this section, we present our method for generating raw estimates of the target's location in the inertial frame. We assume throughout the paper that the target's pixel location in the video image is known. Experimental results are obtained by allowing a user to select the target to be imaged and using a color segmentation algorithm to track the target in the image plane.

\subsection{Coordinate Frames}

The coordinate frames associated with this problem include the inertial frame, the vehicle frame, the body frame, the gimbal frame, and the camera frame. Figures 1 and 2 show schematics of the different coordinate frames. The inertial frame, denoted by $\left(X_{I}, Y_{I}, Z_{I}\right)$, is a fixed frame with $X_{I}$ directed North, $Y_{I}$ directed East, and $Z_{I}$ directed toward the center of the earth. The vehicle frame, denoted by $\left(X_{v}, Y_{v}, Z_{v}\right)$, is oriented identically to the inertial frame but its origin is at the vehicle center of mass. The body frame, denoted by $\left(X_{b}, Y_{b}, Z_{b}\right)$ also originates at the center of mass but is fixed in the vehicle with $X_{b}$ pointing out the nose, $Y_{b}$ pointing out the right wing, and $Z_{b}$ pointing out the belly. As shown in Figures 1 and 2, the gimbal frame, represented by $\left(X_{g}, Y_{g}, Z_{g}\right)$ originates at the gimbal rotation center and is oriented so that $X_{g}$ points along the optical axis, $Z_{g}$ points down in the image plane, and $Y_{g}$ points right in the image plane. The camera frame, denoted $\left(X_{c}, Y_{c}, Z_{c}\right)$, originates at the optical center with $X_{c}$ pointing up in the image, $Y_{c}$ pointing right in the image plane, and $Z_{c}$ directed along the optical axis.

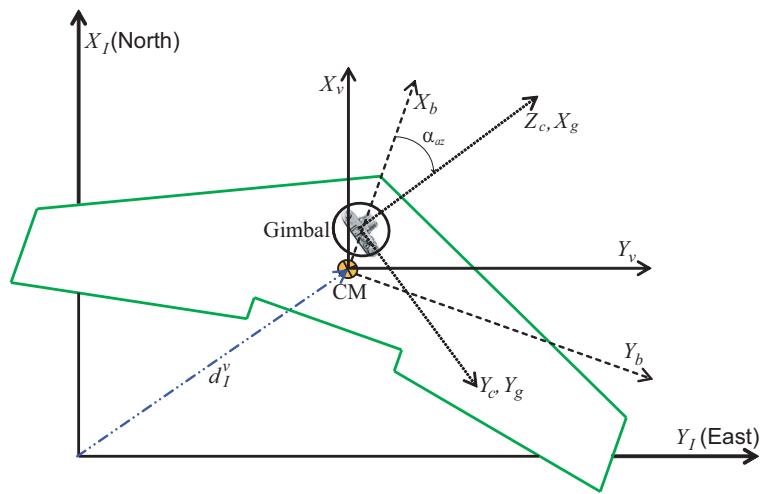

Fig. 1. A graphic showing a lateral view of the coordinate frames. The inertial and vehicle frames are aligned with the world, the body frame is aligned with the airframe, and the gimbal and camera frames are aligned with the camera.

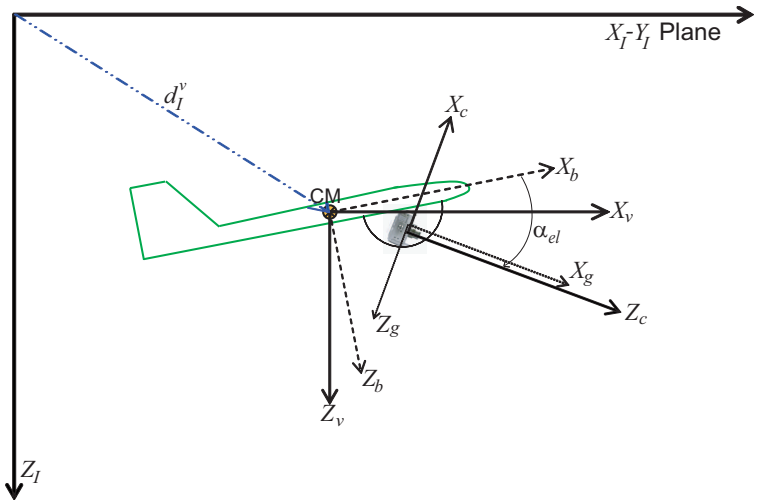

Fig. 2. A graphic showing a longitudinal view of the coordinate frames.

The notation $v^{i}$ implies that vector $v$ is expressed with respect to frame $i$. The rotation matrix and the translation vector from frame $i$ to frame $j$ are denoted by $R_{i}^{j}$ and $d_{i}^{j}$ respectively. The homogeneous transformation matrix from frame $i$ to frame $j$ is given by

$T_{i}^{j}=\left(\begin{array}{cc}R_{i}^{j} & -d_{i}^{j} \\ \mathbf{0} & 1\end{array}\right)$

where $\mathbf{0} \in \mathbb{R}^{3}$ is a row vector of zeros. Note that $d_{i}^{j}$ is resolved in the $j^{\text {th }}$ coordinate frame. The inverse transformation is given by

$T_{j}^{i} \triangleq T_{i}^{j^{-1}}=\left(\begin{array}{cc}R_{i}^{j^{T}} & R_{i}^{j^{T}} \\ \mathbf{0}_{i}^{j} & 1\end{array}\right)$

The transformations used in this papers are defined in Table 1 . The derivation for each of the transformations will be discussed below. 
Table 1. Homogeneous transformation matrices.

\begin{tabular}{c|l}
\hline Transformation & Description \\
\hline$T_{I}^{v}$ & Inertial to MAV Vehicle frame \\
$T_{v}^{b}$ & MAV Vehicle to MAV Body frame \\
$T_{b}^{g}$ & MAV Body to Gimbal frame \\
$T_{g}^{c}$ & Gimbal to Camera frame
\end{tabular}

\subsection{Transformation from the inertial to the vehicle frame.}

The transformation from the inertial to the vehicle frame is a simple translation. Therefore $T_{I}^{v}$ is given by

$$
\begin{aligned}
T_{I}^{v} & =\left[\begin{array}{cc}
I & -d_{I}^{v} \\
0 & 1
\end{array}\right], \quad \text { where } \\
d_{I}^{v} & =\left[\begin{array}{c}
x_{\mathrm{MAV}} \\
y_{\mathrm{MAV}} \\
-h_{\mathrm{MAV}}
\end{array}\right],
\end{aligned}
$$

and where $x_{\mathrm{MAV}}$ and $y_{\mathrm{MAV}}$ represent the North and East location of the MAV as measured by its GPS sensor, and $h_{\mathrm{MAV}}$ represents the MAV's altitude as measured by a calibrated, on-board barometric pressure sensor.

\subsection{Transformation from the vehicle to the body frame.}

The transformation from the vehicle frame to the MAV body frame, $T_{v}^{b}$, consists of a rotation based on measurements of Euler angles. If $\phi, \theta$ and $\psi$ represent the MAV's roll, pitch and heading angles in radians, then the transformation is given by

$$
\begin{aligned}
T_{v}^{b} & =\left[\begin{array}{cc}
R_{v}^{b} & 0 \\
0 & 1
\end{array}\right], \quad \text { where } \\
R_{v}^{b} & =\left[\begin{array}{ccc}
c_{\theta} c_{\psi} & c_{\theta} s_{\psi} & -s_{\theta} \\
s_{\phi} s_{\theta} c_{\psi}-c_{\phi} s_{\psi} & s_{\phi} s_{\theta} s_{\psi}+c_{\phi} c_{\psi} & s_{\phi} c_{\theta} \\
c_{\phi} s_{\theta} c_{\psi}+s_{\phi} s_{\psi} & c_{\phi} s_{\theta} s_{\psi}-s_{\phi} c_{\psi} & c_{\phi} c_{\theta}
\end{array}\right]
\end{aligned}
$$

where $c_{\varphi} \triangleq \cos \varphi$ and $\sin _{\varphi} \triangleq \sin \varphi$. On our platform, the Euler angles are estimated by a two stage Kalman filter as described in Eldredge (2006). The Kalman filter uses rate gyros for the propagation model, and accelerometers for the measurement update.

\subsection{Transformation from the body to the gimbal frame.}

The transformation from the MAV body to the gimbal frame, $T_{b}^{g}$, will depend on the location of the MAV's center of mass with respect to the gimbal's rotation center. This vector, denoted by $d_{b}^{g}$, is resolved in the gimbal frame. $T_{b}^{g}$ will also depend on the rotation that aligns the gimbal's coordinate frame with the MAV's body frame. This rotation is denoted $R_{b}^{g}$ and requires measurements of the camera's azimuth and elevation angles. Let $\alpha_{a z}$ denotes the azimuth angle of rotation about $Z_{g}$, and $\alpha_{e l}$ the elevation angle of rotation about $Y_{g}$, after $\alpha_{a z}$. Both $\alpha_{a z}$ and $\alpha_{e l}$ can be deduced from the gimbal servo commands. The transformation is given by

$$
\begin{aligned}
T_{b}^{g} & =\left[\begin{array}{cc}
R_{b}^{g} & -d_{b}^{g} \\
0 & 1
\end{array}\right], \quad \text { where } \\
R_{b}^{g} & =R_{y, \alpha_{e l}} R_{z, \alpha_{a z}} \\
& =\left[\begin{array}{ccc}
c_{e l} & 0 & s_{e l} \\
0 & 1 & 0 \\
-s_{e l} & 0 & c_{e l}
\end{array}\right]\left[\begin{array}{ccc}
c_{a z} & s_{a z} & 0 \\
-s_{a z} & c_{a z} & 0 \\
0 & 0 & 1
\end{array}\right] \\
& =\left[\begin{array}{ccc}
c_{e l} c_{a z} & c_{e l} s_{a z} & s_{e l} \\
-s_{e l} & c_{a z} & 0 \\
-s_{e l} c_{a z} & -s_{e l} s_{a z} & c_{e l}
\end{array}\right] .
\end{aligned}
$$

\subsection{Transformation from the gimbal to the camera frame.}

The transformation from gimbal to camera reference frames, $T_{g}^{c}$, depends on the vector $d_{g}^{c}$, which describes the location of the gimbal's rotation center relative to the camera's optical center and is resolved in the camera's coordinate frame. $T_{g}^{c}$ also depends on a fixed rotation $R_{g}^{c}$, which aligns the camera's coordinate frame with that of the gimbal since we have chosen $X_{c}=-Z_{g}$ and $Z_{c}=X_{g}$. The transformation is given by

$$
\begin{aligned}
T_{g}^{c} & =\left[\begin{array}{cc}
R_{g}^{c} & -d_{g}^{c} \\
0 & 1
\end{array}\right], \text { where } \\
R_{g}^{c} & =\left[\begin{array}{ccc}
0 & 0 & -1 \\
0 & 1 & 0 \\
1 & 0 & 0
\end{array}\right] .
\end{aligned}
$$

\subsection{Camera Model}

A simple projection camera model is shown in Figure 3. The point $q=\left(x_{i p}, y_{i p}, 1,1\right)^{T}$ is the homogeneous projection of the point $p_{\mathrm{obj}}^{c}=\left(p_{x}, p_{y}, p_{z}, 1\right)^{T}$ onto the image plane in pixels, where $p_{\text {obj }}^{c}$ denotes the location of an object $p$ relative to the center of the camera. Trucco and Verri (2002) show that the change from pixels to meters in the image frame is accomplished by

$$
\begin{aligned}
x_{i m} & =\left(-y_{i p}+0_{y}\right) S_{y} \\
y_{i m} & =\left(x_{i p}-0_{x}\right) S_{x},
\end{aligned}
$$

where the units of $\left(x_{i p}, y_{i p}\right)$ are pixels and the units of $\left(x_{i m}, y_{i m}\right)$ are meters. The parameters $0_{x}$ and $0_{y}$ denote the $x$ and $y$ offsets to the center of the image from the upper-left hand corner in pixels, and $S_{x}$ and $S_{y}$ denote the conversion factors from pixels to meters. By similar triangles we get that

$$
\frac{x_{i m}}{f}=\frac{p_{x}}{p_{z}}, \quad \frac{y_{i m}}{f}=\frac{p_{y}}{p_{z}} .
$$

Using Eq. (6), and defining $\lambda \triangleq p_{z}$ we get

$$
\Lambda q=\underbrace{\left[\begin{array}{cccc}
0 & f_{x} & 0_{x} & 0 \\
-f_{y} & 0 & 0_{y} & 0 \\
0 & 0 & 1 & 0 \\
0 & 0 & 0 & 1
\end{array}\right]}_{C} p_{\mathrm{obj}}^{c}
$$




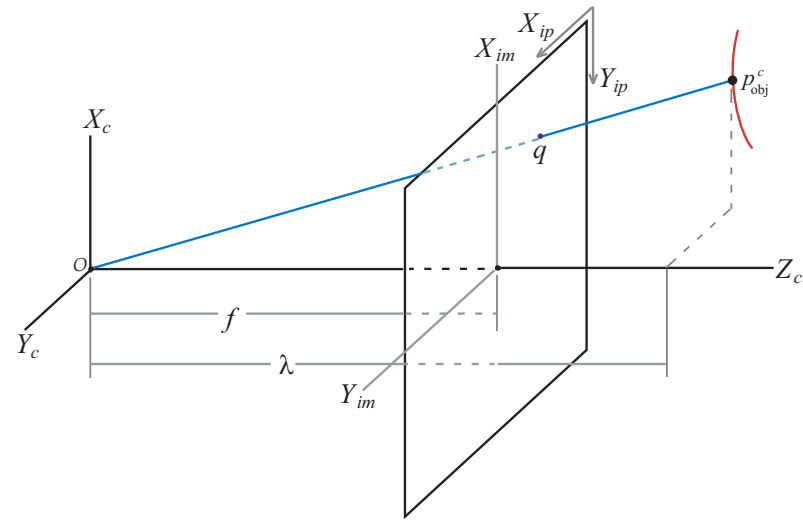

Fig. 3. The coordinate frames associated with the camera. The coordinate frame represented by $\left\{X_{c}, Y_{c}, Z_{c}\right\}$ has origin at the camera center and its elements have units of meters. The frame $\left\{X_{i m}, Y_{i m}, Z_{i m}=Z_{c}-f\right\}$ is centered at the image plane and has units of meters. The frame $\left(X_{i p}, Y_{i p}\right)$ is centered in the upper left hand corner of the image and has units of pixels.

where $f_{x} \triangleq \frac{f}{S_{x}}, f_{y} \triangleq \frac{f}{S_{y}}$, and $\Lambda=\left(\begin{array}{cc}\lambda I & 0 \\ 0 & 1\end{array}\right)$. The matrix $C$ is known as the calibration matrix.

Our objective is to determine $p_{\mathrm{obj}}^{I}$, the object's position in the inertial frame. Using the homogeneous transformations derived in the previous sections we have

$\Lambda q=C p_{\mathrm{obj}}^{c}=C T_{g}^{c} T_{b}^{g} T_{v}^{b} T_{I}^{b} p_{\mathrm{obj}}^{I}$.

Solving for $p_{\text {obj }}^{I}$ gives

$p_{\mathrm{obj}}^{I}=\left[C T_{g}^{c} T_{b}^{g} T_{v}^{b} T_{I}^{v}\right]^{-1} \Lambda q$.

Therefore, $p_{\text {obj }}^{I}$ can be determined when $\lambda$ is known.

\subsection{Image Depth}

The image depth $\lambda$ refers to the distance along the camera's optical axis to the object of interest in the image (Ma et al., 2003). In this paper we describe a technique for estimating $\lambda$ based on a flat earth assumption. A similar technique can be used if a terrain map is available.

Let $p_{\mathrm{cc}}$ be the location of the camera's optical center. If $p_{\mathrm{cc}}$ is resolved in the camera frame we have $p_{\mathrm{cc}}^{c}=(0,0,0,1)^{T}$. Therefore, resolving in the inertial frame gives

$p_{\mathrm{cc}}^{I}=\left(\begin{array}{c}x_{\mathrm{cc}}^{I} \\ y_{\mathrm{cc}}^{I} \\ z_{\mathrm{cc}}^{I} \\ 1\end{array}\right)=\left[T_{g}^{c} T_{b}^{g} T_{v}^{b} T_{I}^{v}\right]^{-1}\left(\begin{array}{l}0 \\ 0 \\ 0 \\ 1\end{array}\right)$.

Figure 4 also shows the location $q=\left[\begin{array}{llll}x_{i p} & y_{i p} & 1 & 1\end{array}\right]^{T}$. Define $q_{\mathrm{obj}}^{I}$ as $q$ resolved in the inertial frame, i.e.,

$q_{\mathrm{obj}}^{I}=\left(\begin{array}{c}x_{\mathrm{obj}}^{I} \\ y_{\mathrm{obj}}^{I} \\ z_{\mathrm{obj}}^{I} \\ 1\end{array}\right)=\left[C T_{g}^{c} T_{b}^{g} T_{v}^{b} T_{I}^{v}\right]^{-1} q$.

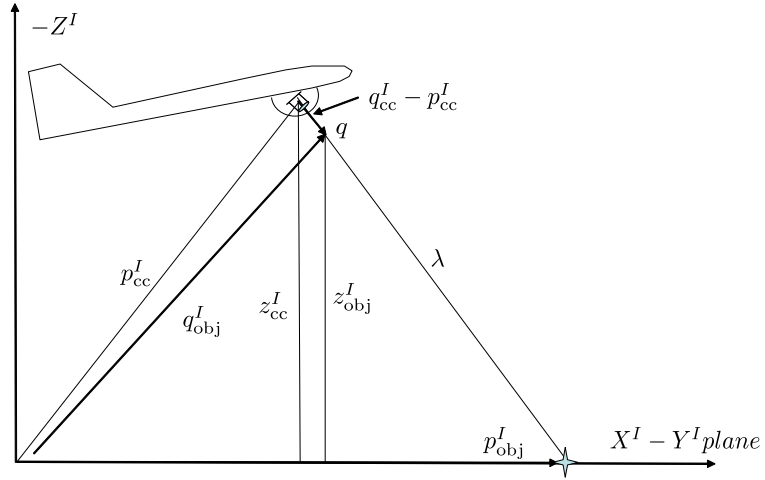

Fig. 4. The range to the target $\lambda$, is estimated using a flat earth model and knowledge of the location and orientation of the MAV and its camera system.

Note from Figure 4 that the flat earth model implies that the relationship between the $z$-components of $q_{\mathrm{obj}}^{I}$ and $p_{\mathrm{cc}}^{I}$ is given by

$0=z_{\mathrm{cc}}^{I}+\lambda\left(z_{\mathrm{obj}}^{I}-z_{\mathrm{cc}}^{I}\right)$.

If a terrain model is known, the zero on the left-hand side of (11) would be modified to reflect the altitude at the point where the optical axis intersects the terrain. Since both $z_{\mathrm{cc}}^{I}$ and $z_{\mathrm{obj}}^{I}$ are known from (9) and (10) respectively, $\lambda$ can be computed as

$\lambda=\frac{z_{\mathrm{cc}}^{I}}{z_{\mathrm{cc}}^{I}-z_{\mathrm{obj}}^{I}}$.

Since the inertial Z-axis, $Z_{I}$, is defined positive toward the center of the earth, $z_{\mathrm{cc}}^{I}$ will be negative for flight altitudes greater than the calibrated zero. Thus, (12) yields a positive value for $\lambda$, as expected.

\subsection{Target Location}

Given $\lambda$, the inertial location of the object is given by

$\begin{aligned} p_{\text {obj }}^{I} & =\left[C T_{g}^{c} T_{b}^{g} T_{v}^{b} T_{I}^{v}\right]^{-1} \Lambda q \\ & =T_{v}^{I} T_{b}^{v} T_{g}^{b} T_{c}^{g} C^{-1} \Lambda q,\end{aligned}$

or equivalently, in the more computationally efficient form

$\bar{p}_{\mathrm{obj}}^{I}=\bar{p}_{\mathrm{cc}}^{I}+\lambda\left(\bar{q}_{\mathrm{obj}}^{I}-\bar{p}_{\mathrm{cc}}^{I}\right)$,

where $\bar{p}$ represents the first three elements or $p$.

Using these equations, we can estimate the geo-location of a target using the telemetry data from the MAV and a time-synchronized video frame containing the target. Unfortunately, every term on the right-hand side of Eq. (13) is computed using measured (i.e. noisy and biased) data. In particular, the transformation matrices $(T)$ and $\Lambda$ are computed using sensor readings, which for MAVs are typically low grade. In the next section we discuss the effects of low quality sensors on the estimation error and introduce four techniques that can be used to reduce the error. 


\section{Enhancing the Geo-location Accuracy}

Sensor noise and uncertainty in the MAV geometry introduces error in the geo-location estimate provided by Eq. (13). Figure 5 shows the results of a flight test using the MAV system described in Section 4.1. The MAV was commanded to orbit the target location and a color segmentation algorithm was used to track the target location in the image. The error (in meters) of the raw estimates of the geo-location of the target are shown in Figure 5. The raw estimates have errors that range from 20 to 40 meters.

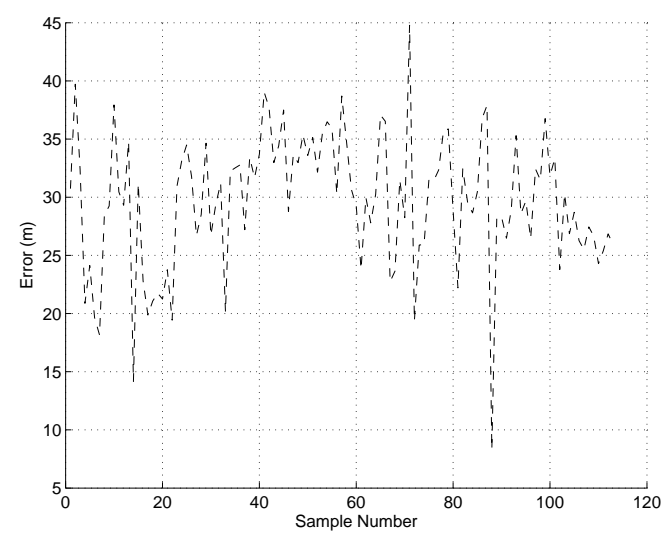

Fig. 5. The error, in meters, of raw geo-location estimates obtained by using Eq. (13). Sensor noise and geometric uncertainties result in estimation errors of 20 to 40 meters.

The primary contribution of this paper is to propose four techniques for enhancing the accuracy of the geo-location estimate. These techniques include: (1) recursive least squares filtering, (2) bias estimation, (3) flight path selection, and (4) wind estimation. Each technique is discussed in more detail below.

\subsection{Recursive Least Squares}

As shown in Figure 5, there is significant noise in the estimation error. In this paper, we assume that the target location is stationary. Therefore, a well known technique to remove the estimation error is to use a recursive least squares (RLS) filter (Moon and Stirling, 2000). The RLS filter minimizes the average squared error of the estimate using an algorithm that only requires a scalar division at each step and is therefore suitable for on-line implementation.

The result of using the RLS filter on the data shown in Figure 5 is shown in Figure 6. Note that the RLS filter quickly converges to an error of approximately five meters. While the improvement in geo-location accuracy is significant, it will be shown in the following three sections that it is possible to further improve the geo-location accuracy by exploiting the structure inherent in the problem.

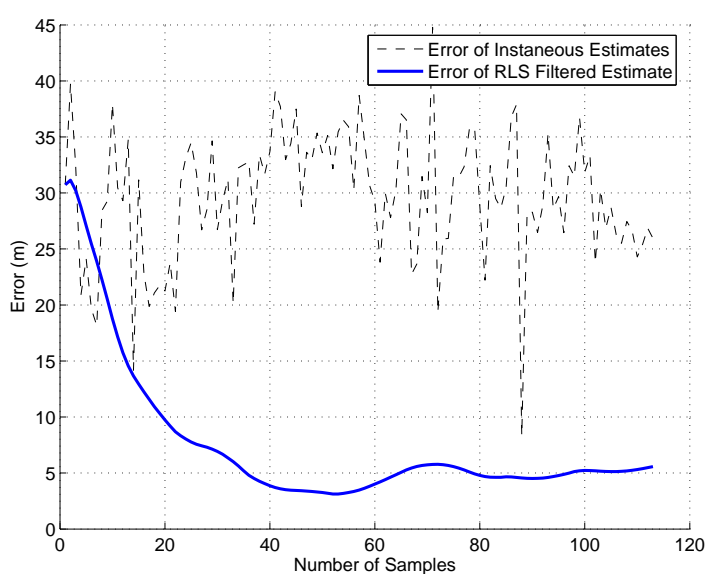

Fig. 6. Result of using the RLS algorithm. The error in the geolocation estimate decreases from 20 to $40 \mathrm{~m}$ to approximately 5 meters.

\subsection{Bias Estimation}

The sensor noise and the geometric uncertainties introduce both zero-mean noise and a constant bias. While the RLS algorithm is effective at removing the zero-mean noise, it is not effective at removing the bias. The geo-location error is particularly sensitive to biases in the roll and the gimbal azimuth measurement, as well as the relative orientation of the gimbal. Although bias errors can be mitigated by advanced attitude estimation schemes and precise mounting and calibration of the camera gimbal, it is impossible to totally remove these bias errors.

Fortunately, by executing a loiter pattern around a specific object, the biases and zero-mean noise can be easily distinguished. Because the bias errors are uncorrelated with respect to position along the desired flight path, and the flight path is symmetric about the target, the bias errors result in geolocation estimates that are also symmetric about the target. For the case of a circular flight path centered at the target, this results in the localization estimates forming a ring around the desired target, as shown in Figure 7.

If the biases are removed from the localization estimates, the geo-location errors would collapse to a 2-D Gaussian distribution centered at the object, as shown in Figure 8. The covariance of the distribution would be a function of the zeromean noise on raw attitude estimates and the selected radius and altitude of the loiter trajectory.

Since the biases may change from flight to flight, an online learning algorithm was developed to estimate and remove them. The algorithm exploits the observation that biases add a ring-like structure to the location estimates, effectively increasing the variance of the estimates. Therefore, if the flight path is a circular orbit about the target and the bias errors are uncorrelated with position along the flight path, then the distribution of location estimates with the smallest variance will be obtained from the unbiased estimate of the target location. As a result, the bias estimation problem can be posed as the following optimization problem:

$\min _{\bar{\alpha}_{\mathrm{az}}, \bar{\alpha}_{\mathrm{el}}, \bar{\phi}, \bar{\theta}, \bar{\psi}, \bar{z}} \sigma_{\text {localization }}^{2}\left(\bar{\alpha}_{\mathrm{az}}, \bar{\alpha}\right.$ el $\left., \bar{\phi}, \bar{\theta}, \bar{\psi}, \bar{z}\right)$ 


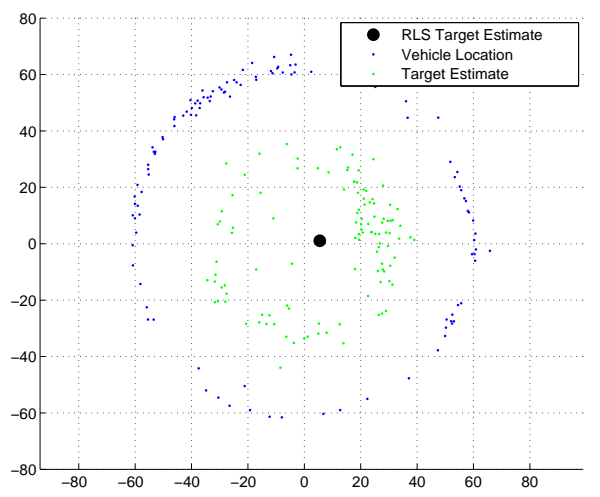

Fig. 7. Localization error before gimbal calibration. The errors in the localization estimates exhibit a circular pattern about the target location due to the biases introduced by imprecisely calibrated sensors and geometric modeling errors.

where $\bar{\alpha}_{\mathrm{az}}, \bar{\alpha}_{\mathrm{el}}, \bar{\phi}, \bar{\theta}, \bar{\psi}$, and $\bar{z}$ are the biases associated with the measurements of gimbal azimuth, gimbal elevation, roll, pitch, yaw, and altitude, respectively.

For the fixed-wing MAVs used in this paper, the center of mass and the gimbal center are located close to each other. Therefore, as can be seen from Figure 1 the rotation axes for heading $\psi$, and gimbal azimuth angle $\alpha_{\text {az }}$ are nearly aligned, making biases introduced by these quantities, virtually indistinguishable. In an orbit pattern, the gimbal azimuth angle will be close to ninety degrees, which implies that the airframe roll axis and the gimbal elevation axis will be nearly aligned, again making biases introduced by $\phi$ and $\alpha_{\text {el }}$ nearly indistinguishable. Even when the flight path is not an orbit, if the body pitch angle is close to zero, then biases introduced by the roll and heading measurements are indistinguishable from biases introduced by gimbal elevation and azimuth measurements. For the MAVs used in this paper, the angle of attack is approximately five degrees, implying that the pitch angle is close to zero for constant altitude flight patterns. Extensive flight testing has also shown that for certain altitude-orbit radius pairs, the estimation error is not significantly affected by biases in pitch and altitude. Therefore, bias estimation can be reduced to the following optimization problem:

$\min _{\bar{\alpha}_{\mathrm{az}}, \bar{\alpha}_{\mathrm{el}}} \sigma_{\text {localization }}^{2}\left(\bar{\alpha}_{\mathrm{az}}, \bar{\alpha}_{\mathrm{el}}\right)$.

Eq. (16) is solved on-line using a quasi Newton method.

Once the biases have been determined, their effects are removed by using the corrected measurements for $\alpha_{\mathrm{el}}$ and $\alpha_{\mathrm{az}}$ in Eq. (13) to obtain unbiased raw estimates. The effects of bias estimation and correction on the dispersion of raw target estimates can be seen in Figure 8. It is clear from Figure 8 that the ring structure characteristic of bias errors has been dramatically reduced.

\subsection{Flight Path Selection}

With the bias removed, we turn our attention to minimizing the variance of the resulting zero-mean estimation error. The variance is primarily due to noisy estimates of the attitude and

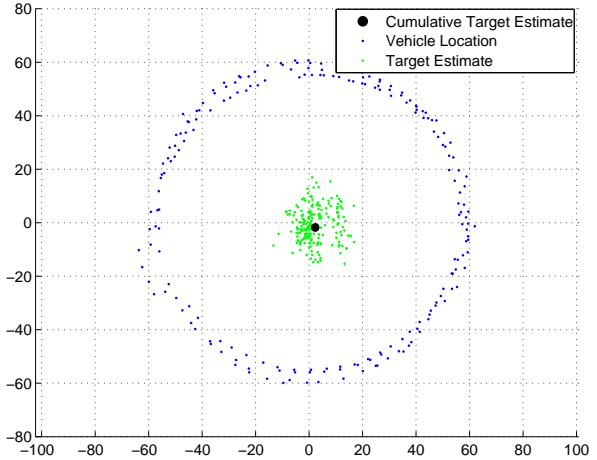

Fig. 8. Localization error after gimbal calibration. The structured bias in the estimates has been removed.

the position of the MAV. Redding (2005) presents a study of the sensitivity of Eq. (13) to errors in attitude and position. The conclusion of that study is that for circular orbits, the geo-location estimate is most sensitive to errors in roll. However, the sensitivity is a strong function of altitude and orbit radius. As shown in Figure 9, as the altitude of the MAV increases, the distance to the target also increases. Therefore, a fixed error in roll, produces a localization error that increases with altitude. On the other hand, Figure 9 illustrates that low altitudes also enhance the error sensitivity since the angle to the target becomes more oblique as altitude decreases. For an identical error in roll, increasingly oblique angles produce a larger localization error.

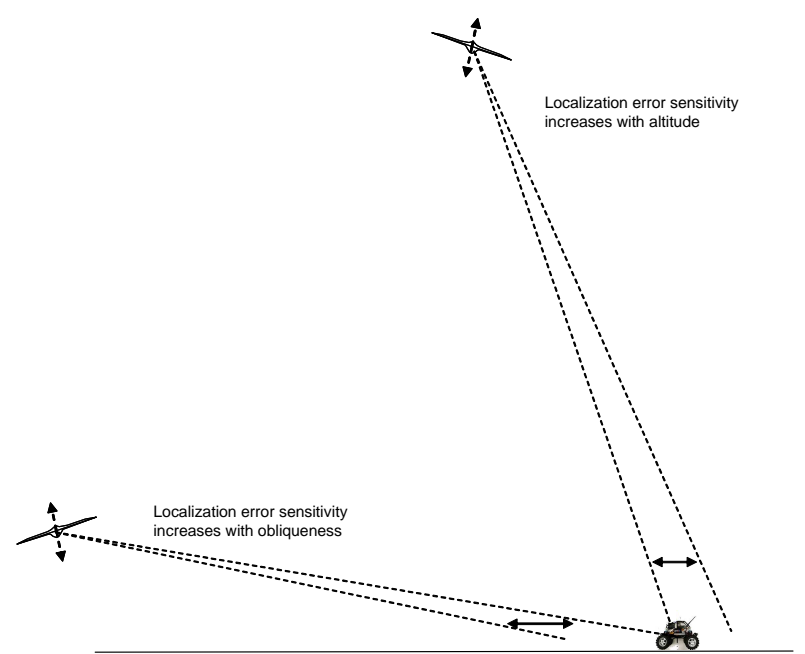

Fig. 9. The sensitivity of the localization error to imprecise attitude estimates, is highly dependent on altitude. At low altitudes, the sensitivity is due to the obliqueness of the angle to the target. At high altitudes, the sensitivity is due to distance from the target.

To explore the relationship between sensitivity to roll attitude and the altitude and orbit radius, consider the simplified situation shown in Figure 10, where we have assumed that the camera is aligned with the right wing and is pointing directly at the target, and that the pitch angle is zero. Therefore, the nominal roll angle is $\phi_{\text {nom }}=\tan ^{-1} \frac{h}{R}$ where $h$ is the altitude 
and $R$ is orbit radius. If the roll angle deviates from the nominal by $\delta \phi$, Eq. (13) will indicate a geo-location of $R-\delta R$ instead of $R$. For the simplified geometry shown in Figure 10

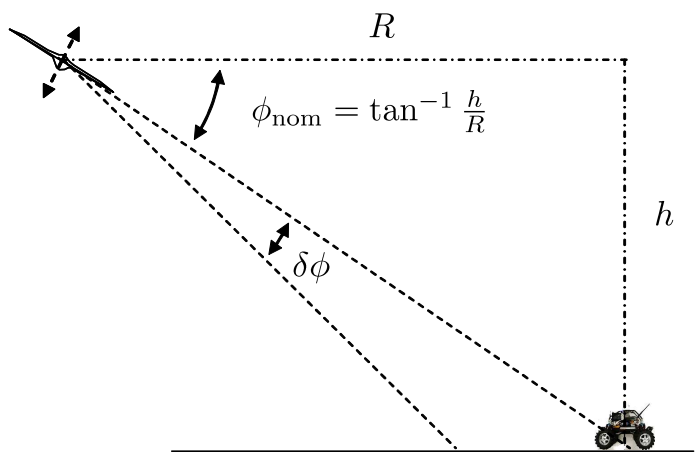

$\delta R$

Fig. 10. Simplified geometry used to derive an expression for the sensitivity of the localization error to the roll angle as a function of the orbit altitude and radius.

we have that

$R-\delta R=\frac{h}{\tan (\phi+\delta \phi)}$.

Therefore, using the relations $\tan (A+B)=\frac{\tan (A)+\tan B}{1-\tan (A) \tan (B)}$ and $\tan \phi=\frac{h}{R}$, we obtain

$$
\begin{aligned}
\delta R & =R-\frac{h}{\tan (\phi+\delta \phi)} \\
& =R-h \frac{1-\tan \phi \tan \delta \phi}{\tan \phi+\tan \delta \phi} \\
& =R-h \frac{R-h \tan \delta \phi}{h+R \tan \delta \phi} \\
& =\frac{\left(R^{2}+h^{2}\right) \tan \delta \phi}{h+R \tan \delta \phi} .
\end{aligned}
$$

Figure 11 shows a plot of Eq. (17) as a function of $h$ for $\delta \phi=5$ degrees and $R=100$ meters. It is clear that for a fixed radius, there is an optimal altitude that minimizes the sensitivity of the localization error to deviations in the roll attitude. The optimal altitude is found by differentiating Eq. (17) with respect to $h$ and solving for the unique minimizer:

$h^{*}=R\left(\frac{1-\sin \delta \phi}{\cos \delta \phi}\right)$.

Therefore, if we have an estimate for the average (or maximum) roll attitude error, and there is a desired orbit radius, e.g., the minimum turn radius, then Eq. (18) indicates the appropriate altitude for minimizing the sensitivity of the geolocation estimate to errors in the roll attitude measurement.

As an alternative, the computer vision algorithm may require a specific number of pixels-on-target to effectively track the target. In order to talk more generally about the notion of pixels-on-target, we define the pixel density to be the number of pixels imaging a square meter of area on the ground. Let $\mu$

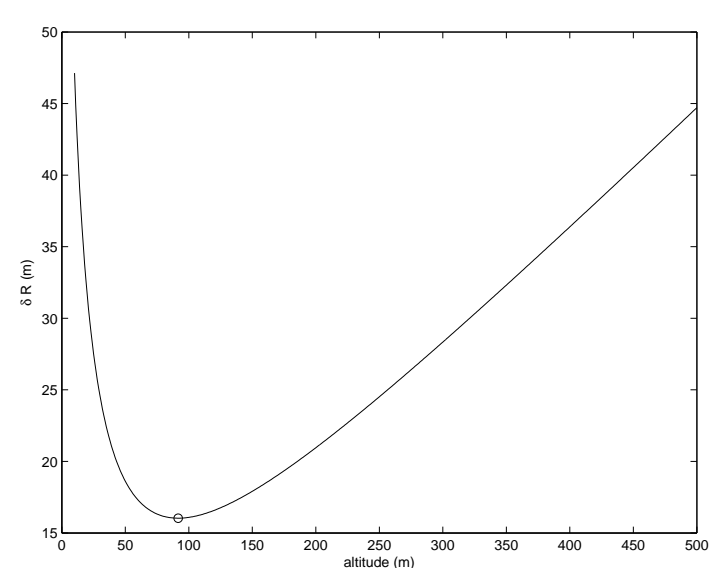

Fig. 11. A plot of the geo-location error as a function of altitude for a fixed radius and a fixed roll attitude error. The optimal altitude $h^{*}$ in indicated by a circle.

denote pixel density in units of pixels per meters squared. If $\eta$ is the field of view of the lens then the area imaged by the camera can be computed by referencing Figure 12 . The total area is given by

$$
\begin{aligned}
\text { Area } & =\left(d_{1}+d_{2}\right)\left(R_{2}-R_{1}\right) \\
& =\left(R_{2}+R_{1}\right)\left(R_{2}-R_{1}\right) \tan \frac{\eta}{2} \\
& =h^{2}\left(\frac{1}{\tan ^{2}\left(\phi-\frac{\eta}{2}\right)}-\frac{1}{\tan ^{2}\left(\phi+\frac{\eta}{2}\right)}\right) \tan \frac{\eta}{2} \\
& \triangleq h^{2} \mathcal{A} .
\end{aligned}
$$

If $P$ is the number of pixels on the camera, then the pixel density is given by

$\mu=\frac{P}{h^{2} \mathcal{A}}$.

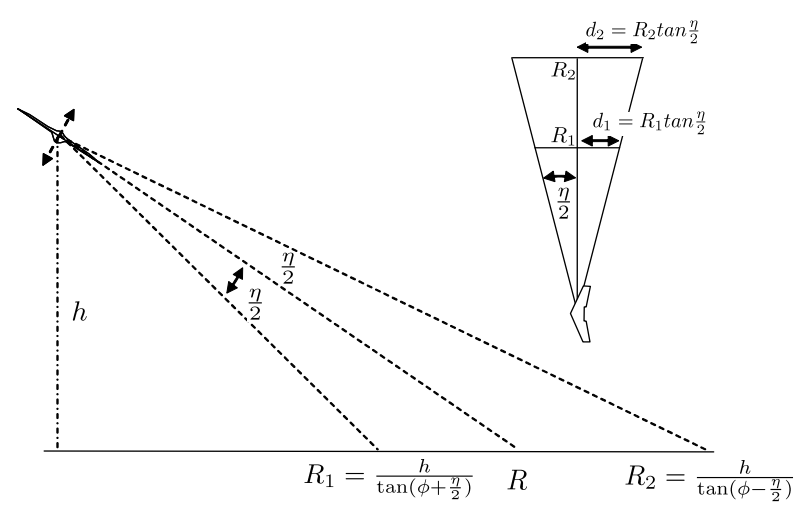

Fig. 12. Assuming a flat earth model, the area imaged by the camera can be computed by knowledge of the roll angle $\phi$ the lens field $\eta$, and the altitude $h$.

Suppose that the computer vision algorithm requires a desired pixel density of $\mu^{d}$, then using Eqs. (19) and (18) we get 
that the optimal altitude and orbit radius are given by

$$
\begin{aligned}
h^{*} & =\sqrt{\frac{P}{\mu^{d} \mathcal{A}}} \\
R^{*} & =h^{*}\left(\frac{\cos \delta \phi}{1-\sin \delta \phi}\right) .
\end{aligned}
$$

\subsection{Wind Estimation}

For MAVs, winds that are a significant percentage of the airspeed are almost always present. Therefore, the airframe is typically required to "crab" into the wind, causing the course (direction of travel) to deviate from the heading (direction of the body frame $x$-axis). Since the camera is mounted to the body, the difference between course and heading, if it is not accounted for, will cause significant errors in the geo-location estimates. In this section, the heading angle is denoted by $\psi$ and the course angle will be denoted by $\chi$.

To illustrate the effect of wind, Figure 13 shows the error in the geo-location estimate generated by a simulation of a complete orbit in significant wind. The simulated MAV has an airspeed of $18 \mathrm{~m} / \mathrm{s}$ and the wind is from the East at $9 \mathrm{~m} / \mathrm{s}$. Note that since the MAV must crab right into the wind, the

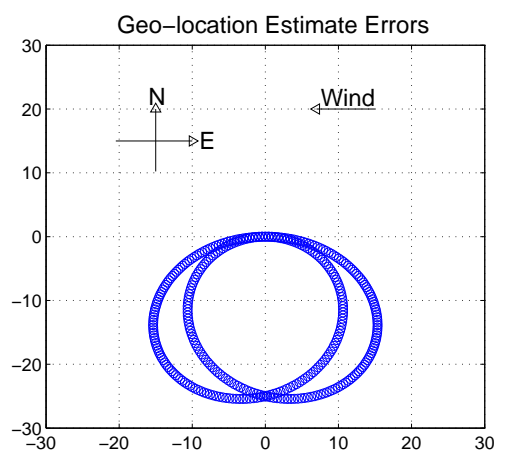

Fig. 13. Effect of wind on geo-location estimates

geo-location errors shown in Figure 13 are significantly biased to the South. We note that wind does not introduce a constant bias in the estimate and can therefore not be removed by the techniques discussed in Section 3.2. To compensate for wind, the direction and magnitude of the wind is estimated on-line from flight data and is used to modify $\psi$ in Eq. (13). We will assume that GPS measurements are available but that the MAV is not equipped with magnetometers.

The relationship between windspeed, groundspeed, and airspeed is illustrated in Figure 14, where $V_{w}$ is the windspeed, $V_{g}$ is the groundspeed, $V_{a}$ is the airspeed, and $\xi$ is the wind direction, and can be expressed as

$V_{g}=V_{a} \cos (\psi-\chi)+V_{w} \cos (\xi-\chi)$.

The GPS sensor measures $V_{g}$ and $\chi$, and a differential pressure sensor can be used to measure $V_{a}$. The law of cosines

$\cos (A)=\frac{-a^{2}+b^{2}+c^{2}}{2 b c}$,

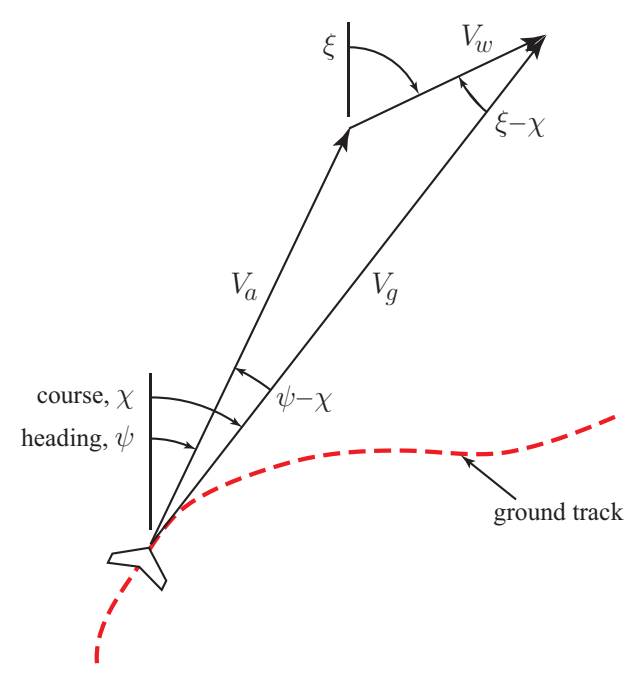

Fig. 14. Relationship between ground, air, and wind velocities.

can be used to remove $\psi$ from Eq. (20), resulting in the expression

$V_{g}=\left(\frac{V_{g}^{2}+V_{a}^{2}-V_{w}^{2}}{2 V_{g}}\right)+V_{w} \cos (\xi-\chi)$.

Rearranging we obtain

$V_{g}^{2}-V_{a}^{2}+V_{w}^{2}-2 V_{g} V_{w} \cos (\xi-\chi)=0$.

To estimate $V_{w}$ and $\xi$ we collect on-line measurements of $V_{g}$, $V_{a}$, and $\chi$ and use a quasi-Newton nonlinear equation solver to minimize the objective function

$\sum_{i=0}^{n}\left(V_{g_{i}}^{2}-V_{a_{i}}^{2}+V_{w}^{2}-2 V_{g_{i}} V_{w} \cos \left(\xi-\chi_{i}\right)\right)^{2}$,

where the index $i$ denotes a measurement sample.

To quantify the effectiveness of our wind estimation scheme, we flew a MAV in windy conditions in an orbit pattern. Since we do not have the instrumentation to measure true wind speed at the elevations that the MAV is flying (100-200 meters), to measure the accuracy of our wind estimation method, we used the estimated windspeed and the measured airspeed to estimate ground speed, and compared the estimate with the measured GPS ground speed. Figure 15 shows actual flight data recorded while the MAV was flying in winds of approximately $9 \mathrm{~m} / \mathrm{s}$ from the north. Figure 15 shows the efficacy of our method by plotting the raw airspeed measurements, as well as the estimated airspeed using the estimated windspeed and the GPS measured groundspeed. Results demonstrating the efficacy of the wind correction scheme for geo-location are discussed in Section 4.2.

The wind estimation scheme discussed in this section estimates a constant wind and does not account for gusts. On 


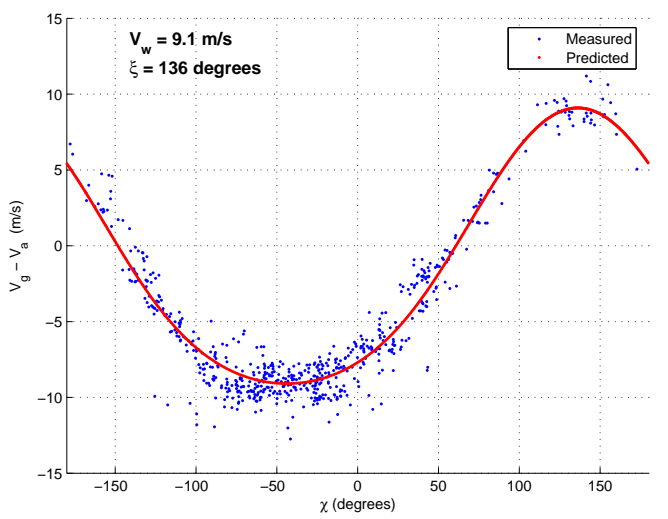

Fig. 15. Wind solution for a dataset taken in high-wind conditions $V_{w} \approx 9 \mathrm{~m} / \mathrm{s}$

the other hand, flight test data suggest that the gusts are essentially normally distributed about the constant wind and are therefore removed by the RLS filter.

\section{Results}

\subsection{Hardware Testbed}

BYU has developed a reliable and robust platform for testing unmanned air vehicles (Beard et al., 2005). Figure 16 shows the key elements of the testbed. The first frame shows the Procerus ${ }^{1}$ Kestrel autopilot (originally developed at BYU) which which is equipped with a Rabbit $340029 \mathrm{MHz}$ processor, rate gyros, accelerometers, absolute and differential pressure sensors. The autopilot measures $3.8 \times 5.1 \times 1.9 \mathrm{~cm}$ and weighs 17 grams.

The second frame in Figure 16 shows the airframe used for the flight tests reported in this paper. The airframe is a flying wing with expanded payload bay and servo-driven elevons designed by the BYU Magicc Lab. It has a wingspan of $152 \mathrm{~cm}$, a length of $58 \mathrm{~cm}$, and a width of $12 \mathrm{~cm}$. It weighs $1.1 \mathrm{~kg}$ unloaded and $2.0 \mathrm{~kg}$ fully loaded. It is propelled by a brushless electric motor which uses an electronic speed control and is fueled by four multi-cell lithium polymer batteries. Typical speeds for the aircraft are between 15 and 20 meters per second (33 and 45 miles per hour). Maximum flight time for this aircraft is between 1 and 2 hours depending on external conditions and the mission it is required to fly.

The third frame in Figure 16 shows the ground station components. A laptop runs the Virtual Cockpit software that interfaces through a communication box to the MAV. An RC transmitter is used as a stand-by fail-safe mechanism to ensure safe operations.

The gimbal and camera used for this paper are shown in Figure 17. The gimbal was designed and constructed by the BYU Magicc Lab. It weighs 150 grams, and has a range of motion of 135 degrees in azimuth (at 333 degrees/sec) and 120 degrees in elevation (at 260 degrees/sec). The camera is a Panasonic KX-141 with 480 lines of resolution. The field of view of the lens is 30 degrees.

\footnotetext{
${ }^{1} \mathrm{http}: / /$ procerusuav.com/
}

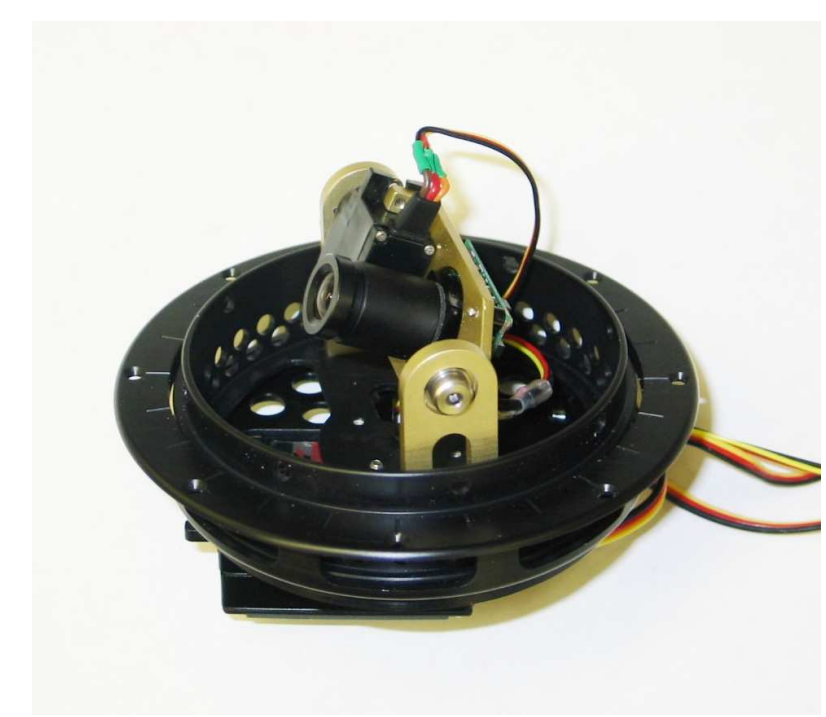

Fig. 17. The gimbal and camera used for the results in the paper are shown unmounted from the MAV and without its protective dome.

\subsection{Geo-location Accuracy}

Using the MAV system described above, in conjunction with the geo-location techniques described in this paper, we have repeatedly ( 15 to 20 experiments in a variety of weather conditions) geo-located well defined visual objects, with errors ranging between 2 and 4 meters. (The true value of the target is measured using the same commercial grade GPS receiver used on the MAV. Note that the geo-location techniques discussed in this paper do not remove GPS bias. A military grade GPS, or differential GPS would remove this bias.) The results of two particular flight tests are shown in Figures 8 and 18 . The outer blue dots represent the GPS location of the MAV, while the inner green dots are the raw geo-location estimates. All location values are in reference to the true location of the target (as measured by GPS). The flight tests shown in Figure 8 were performed on a day with relatively little wind, while the flight tests shown in Figure 18 were performed in extremely high-wind conditions $(>10 \mathrm{~m} / \mathrm{s})$. Note that the high-wind conditions cause the irregular flight pattern shown in Figure 18. In both Figures, the accuracy of the raw geolocation estimates is typically less than 20 meters, although there are some outliers in the high-wind case. The black dot in the center of the figures represents the final geo-location estimate, and is approximately three meters away from the target in the low-wind case, and two meters away in the high-wind case.

In Figure 19, we show the effects of using the RLS system to derive the final geo-location estimate. In this plot, the $x$-axis denotes different raw estimates of geo-location (typically estimated about three times per second), while the $y$ axis denotes the magnitude of the localization error. The data in this graph corresponds with the low-wind experiment plotted in Figure 8. As illustrated in Figure 19, the raw estimates can be up to $20 \mathrm{~m}$ in error. However, the RLS filtered estimate quickly converges to less than five meters of error. 


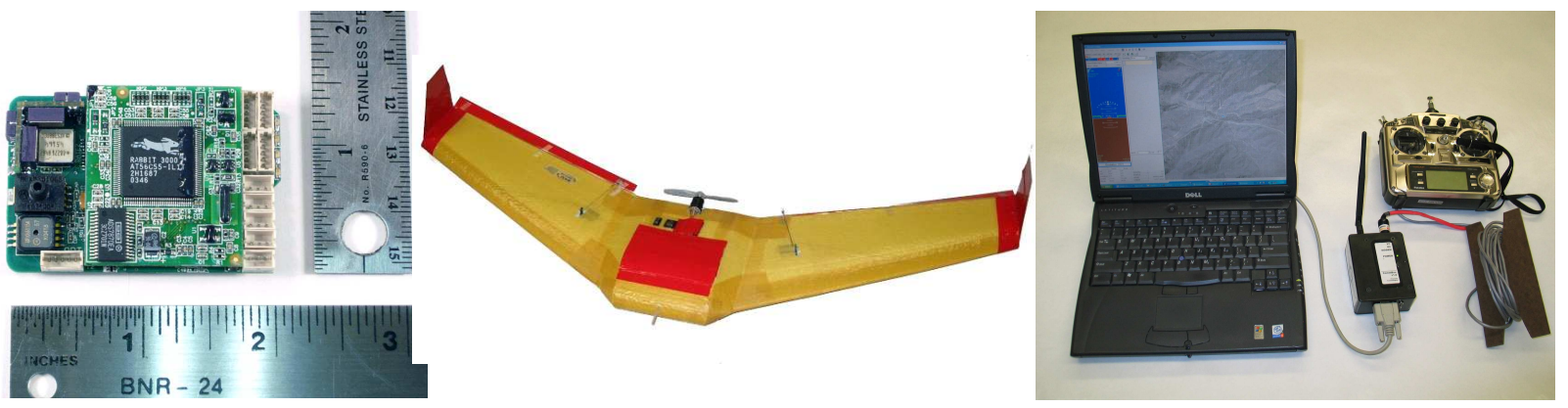

Fig. 16. (a) Procerus' Kestrel autopilot. (b) MAV airframe. (c) Ground station components.

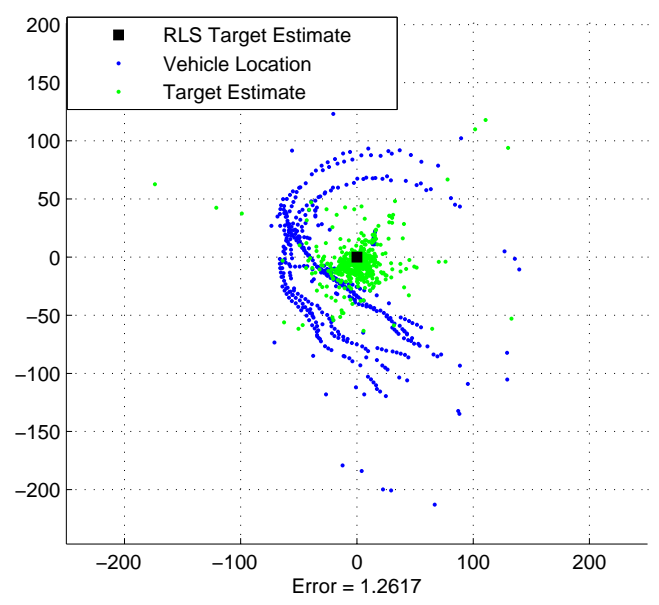

Fig. 18. Localization results in high-wind conditions

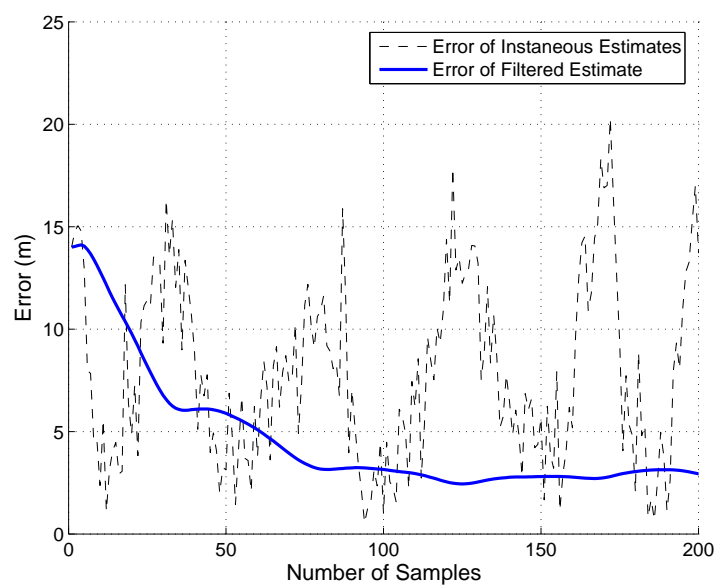

Fig. 19. Efficacy of RLS algorithm

\section{Conclusions}

This paper introduces a system for vision-based target geolocalization from a fixed-wing micro air vehicle. The geometry required to produce raw localization estimates is discussed in detail. The primary contribution of the paper is the description of four key techniques for mitigating the error in the raw estimates. These techniques include RLS filtering, bias estimation, flight path selection, and wind estimation. The al- gorithms were successfully flight tested on a micro air vehicle using Procerus' Kestrel autopilot and a BYU designed gimbal system. Geo-location errors below five meters were repeatedly obtained under a variety of weather conditions. Throughout the paper we have assumed a flat earth model and a stationary target. Future research will include generalizing the techniques to non-flat terrain and to moving ground targets.

Acknowledgements. This work was funded by AFOSR award number FA9550-04-1-0209 and the Utah State Centers of Excellence Program. The authors would like to thank Andrew Eldridge and David Johansen for their assistance in obtaining flight results.

\section{References}

Beard, R., Kingston, D., Quigley, M., Snyder, D., Christiansen, R., Johnson, W., McLain, T., and Goodrich, M. (2005). Autonomous vehicle technologies for small fixed wing UAVs. AIAA Journal of Aerospace Computing, Information, and Communication, 2(1):92-108.

Campbell, M. E. and Wheeler, M. (2006). A vision based geolocation tracking system for uavs. In Proceedings of the AIAA Guidance, Navigation, and Control Conference and Exhibit, Keystone, Colorado. Paper No. AIAA-2006-6246.

Chaimowicz, L., Grocholsky, B., Keller, J. F., Kumar, V., and Taylor, C. J. (2004). Experiments in Multirobot Air-Ground Coordination. In Proceedings of the 2004 International Conference on Robotics and Automation, pages 4053-4058, New Orleans, LA.

Chroust, S. G. and Vincze, M. (2003). Fusion of Vision and Inertial Data for Motion and Structure Estimation. Journal of Robotic Systems, 21:73-83.

Dobrokhodov, V. N., Kaminer, I. I., and Jones, K. D. (2006). Visionbased tracking and motion estimation for moving targets using small uavs. In Proceedings of the AIAA Guidance, Navigation, and Control Conference and Exhibit, Keystone, Colorado. Paper no. AIAA-2006-6606.

Eldredge, A. M. (2006). Improved state estimation for miniature air vehicles. Master's thesis, Brigham Young University.

Frew, E. and Rock, S. (2003). Trajectory Generation for MonocularVision Based Tracking of a Constant-Velocity Target. In Proceedings of the 2003 IEEE International Conference on Robotics and Automation.

Gibbins, D., Roberts, P., and Swierkowski, L. (2004). A video geolocation and image enhancement tool for small unmanned air vehicles (UAVs). In Intelligent Sensors, Sensor Networks and Information Processing Conference, 2004. Proceedings of the 2004, pages 469-473.

Kumar, R., Samarasekera, S., Hsu, S., and Hanna, K. (2000). Registration of highly-oblique and zoomed in aerial video to reference 
imagery. In Proceedings of the IEEE Computer Society Computer Vision and Pattern Recognition Conference, Barcelona, Spain.

Kumar, R., Sawhney, H., Asmuth, J., Pope, A., and Hsu, S. (1998). Registration of video to geo-referenced imagery. In Pattern Recognition, 1998. Proceedings. Fourteenth International Conference on, volume 2, pages 1393-1400.

Ma, Y., Soatto, S., Kosecka, J., and Sastry, S. S. (2003). An Invitation to 3-D Vision From Images to Geometric Models. SpringerVerlag, New York, USA.

Moon, T. K. and Stirling, W. C. (2000). Mathematical Methods and Algorithms. Prentice Hall, Englewood Cliffs, New Jersey.

Office of the Secretary of Defense, editor (2002). Unmanned Aerial Vehicles Roadmap 2002-2027. United States Government, Washington DC, USA.

Redding, J., McLain, T. W., Beard, R. W., and Taylor, C. (2006). Vision-based target localization from a fixed-wing miniature air vehicle. In Proceedings of the American Control Conference, pages 2862-2867, Minneapolis, Minnesota.

Redding, J. D. (2005). Vision based target localization from a small fixed-wing unmanned air vehicle. Master's thesis, Brigham Young University, Provo, Utah 84602.

Rysdyk, R. (2003). UAV Path Following for Constant Line-of-sight. In 2nd AIAA "Unmanned Unlimited" Systems, Technologies and Operations Aerospace, Land and Sea Conference.

Saeedi, P., Lowe, D. G., and Lawrence, P. D. (2003). 3D Localization and Tracking in Unknown Environments. In Proceedings of the IEEE Conference on Robotics and Automation, volume 1, pages 1297-1303.

Schultz, H., Hanson, A., Riseman, E., Stolle, F., and Zhu, Z. (2000). A system for real-time generation of geo-referenced terrain models. SPIE Enabling Technologies for Law Enforcement, Boston, $M A$ (USA).

Stolle, S. and Rysdyk, R. (2003). Flight Path Following Guidance for Unmanned Air Vehicles with Pan-Tilt Camera for Target Observation. In 22nd Digital Avionics Systems Conference.

Trucco, E. and Verri, A. (2002). Introductory Techniques for 3-D Computer Vision. Prentice-Hall, New Jersey, USA.

Vidal, R. and Sastry, S. (2002). Vision-Based Detection of Autonomous Vehicles for Pursuit-Evasion Games. In IFAC World Congress on Automatic Control, Barcelona, Spain.

Wang, I., Dobrokhodov, V., Kaminer, I., and Jones, K. (2005). On Vision-Based Target Tracking and Range Estimation for Small UAVs. In 2005 AIAA Guidance, Navigation, and Control Conference and Exhibit, pages 1-11.

Whang, I. H., Dobrokhodov, V. N., Kaminer, I. I., and Jones, K. D. (2005). On vision-based tracking and range estimation for small uavs. In Proceedings of the AIAA Guidance, Navigation, and Control Conference and Exhibit, San Francisco, CA. Paper no. AIAA-2005-6401. 